\title{
Mid-infrared Type-II InAs/GaSb Superlattice Photodiodes Fabricated on InP Substrates
}

\author{
Kohei Miura*, Yasuhiro Iguchi, Tsukuru Katsuyama, \\ Yuichi Kawamura ${ }^{1}$, Junpei Murooka ${ }^{2}$, Haruyoshi Katayama ${ }^{2}$, \\ Shota Sugano ${ }^{3}$, Tomoko Takekawa ${ }^{3}$ and Masafumi Kimata ${ }^{3}$ \\ Transmission Devices R\&D Laboratories, Sumitomo Electric Industries, Ltd., \\ 1, Taya-cho, Sakae-ku, Yokohama, Kanagawa 244-8588, Japan \\ ${ }^{1}$ Graduate School of Engineering, Osaka Prefecture University, \\ 1-2 Gakuen-cho, Naka-ku, Sakai, Osaka 599-8570, Japan \\ ${ }^{2}$ Earth Observation Research Center, Japan Aerospace Exploration Agency, \\ 2-1-1 Sengen Tsukuba, Ibaraki 305-0035, Japan \\ ${ }^{3}$ Graduate School of Science and Engineering, Ritsumeikan University, \\ 1-1-1 Noji-higashi, Kusatsu, Shiga 525-8577, Japan \\ (Received November 28, 2013; accepted February 17, 2014)
}

Key words: GaSb, InAs, type-II superlattice, InP, mid-infrared photodiode

Type-II InAs/GaSb superlattices (SLs) are attractive material systems for mid-infrared photodiodes (PDs) with higher detectivities than the conventional $\mathrm{HgCdTe}$. GaSb substrates are generally used for the epitaxial growth of these type-II SLs. However, in the case of back-illuminated PDs, the GaSb substrate should be nearly removed because of its strong absorption of mid-infrared light. In this study, infrared PDs with a cut-off wavelength of $7 \mu \mathrm{m}$ were fabricated for the first time by using the 100 period of InAs/ $\mathrm{GaSb}$ SLs grown on an InP substrate, which has much less absorption of mid-infrared light. The dark current density at the reverse bias of $-0.1 \mathrm{~V}$ and at a temperature of 112 $\mathrm{K}$ was $33.0 \mathrm{~mA} / \mathrm{cm}^{2}$. External quantum efficiency over $10 \%$ at the wavelength of $5 \mu \mathrm{m}$ was obtained.

\section{Introduction}

Mid-infrared photodiodes (PDs) with a cut-off wavelength over $3 \mu \mathrm{m}$ are used for gas sensing, thermal detection and so forth. PDs made of $\mathrm{HgCdTe}$ have been widely used for highly sensitive and high-speed operations. Recently, type-II InAs/GaSb superlattices (SLs) have been regarded as promising alternative material systems because of the easy controllability of their bandgap energy and the prediction of lower dark currents owing to the suppression of Auger recombination. ${ }^{(1,2)}$ Focal plane arrays (FPAs) using InAs/ GaSb SL absorption layers have been reported. ${ }^{(3-6)}$ GaSb substrates are generally used for epitaxial growth. However, in the case of back-illuminated PDs such as FPAs, the

*Corresponding author: e-mail: miura-kouhei@sei.co.jp 
GaSb substrate should be nearly removed because of its large absorption coefficient in the infrared region. ${ }^{(7)}$ Furthermore, the large difference in the thermal expansion coefficient between GaSb and silicon makes the reliability of the FPA poor. The FPA is fabricated by bonding a PD chip to a silicon read-out integrated circuit via bump metals. When the FPA is cooled to an operational temperature lower than $100 \mathrm{~K}$, significant stress is applied to the bonding part due to the difference in the thermal expansion coefficient. The InAs/GaSb SLs grown on GaAs substrates with high transparency in the infrared region are proposed and demonstrated.(7) However, the difference in the thermal expansion coefficient between GaAs and silicon is still large. The GaAs also has the problem that the large lattice mismatch between $\mathrm{GaAs}$ and $\mathrm{GaSb}(7.8 \%)$ makes the epitaxial growth difficult. ${ }^{(8,9)}$

InP substrates are more favorable because of not only a high transparency in the midinfrared region but also a smaller lattice mismatch to the GaSb as listed in Table 1(a). Moreover, the thermal expansion coefficient of InP is closer to that of Si than to those of GaAs and GaSb [Table 1(b)]. Miura et al. have reported that InAs/GaSb SLs with good crystalline and optical qualities were successfully grown on InP substrates. ${ }^{(10)}$ By using a thicker GaSb buffer layer on the InP substrate, the full width at half maximum of X-ray satellite peaks and the photoluminescence intensities were improved. In this study, mid-infrared PDs with InAs/GaSb SL absorption layers grown on InP substrates were successfully fabricated for the first time.

\section{Materials and Methods}

First of all, the absorption coefficient of InP substrates was measured. Absorption coefficient spectra of several substrates are shown in Fig. 1. An Fe-doped InP substrate was proved to have a lower absorption coefficient in the wavelength range of 3-12 $\mu \mathrm{m}$ than an undoped $\mathrm{GaSb}$ substrate and a Te-doped $\mathrm{GaSb}$ substrate, probably due to less free carrier absorption. In this study, we used Fe-doped InP substrates.

Epitaxial growth was performed by the solid-source MBE method. In and Ga metals were used as group III beam sources and supplied by conventional effusion cells. Group

Table 1

Parameters of GaSb, GaAs and InP.

(a) Lattice constant

\begin{tabular}{lccc}
\hline & Lattice constant $(\AA)$ & Lattice mismatch with GaSb & Reference \\
\hline $\mathrm{GaSb}$ & 6.096 & - & 11 \\
$\mathrm{GaAs}$ & 5.653 & $7.8 \%$ & 11 \\
$\mathrm{InP}$ & 5.870 & $3.9 \%$ & 11 \\
\hline
\end{tabular}

(b) Thermal expansion coefficient

\begin{tabular}{lcc}
\hline & Thermal expansion coefficient $\left(10^{-6} \mathrm{~K}^{-1}\right)$ & Reference \\
\hline $\mathrm{Si}$ & 3.34 & 12 \\
$\mathrm{GaSb}$ & 7.74 & 11 \\
$\mathrm{GaAs}$ & 6.86 & 11 \\
$\mathrm{InP}$ & 4.75 & 11 \\
\hline
\end{tabular}




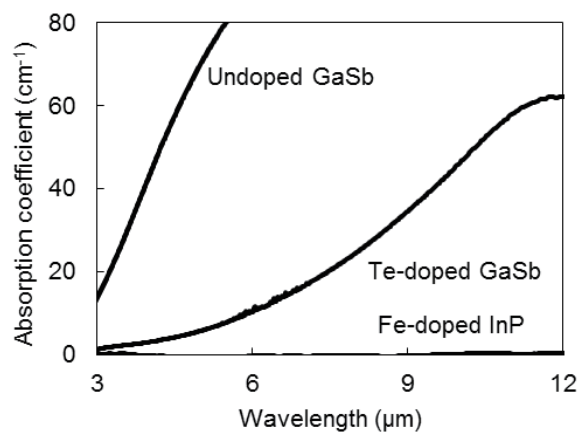

Fig. 1. Absorption coefficient spectra of $\mathrm{InP}$ and GaSb substrates.

$\mathrm{V}$ sources of $\mathrm{As}$ and $\mathrm{Sb}$ were supplied by needle-valve cracking cells. The cracking temperatures were kept at 600 and $800{ }^{\circ} \mathrm{C}$ for $\mathrm{As}$ and $\mathrm{Sb}$, respectively. The substrate temperature was measured using a pyrometer.

Prior to growth, the Fe-doped (100) InP substrate surface was cleaned at $540{ }^{\circ} \mathrm{C}$ under As vapor pressure. An undoped $\mathrm{In}_{0.53} \mathrm{Ga}_{0.47} \mathrm{As}$ layer of $0.15 \mu \mathrm{m}$ thickness and a Be-doped $\mathrm{GaSb}$ buffer layer of $4.5 \mu \mathrm{m}$ thickness were grown on the substrate. The $\mathrm{In}_{0.53} \mathrm{Ga}_{0.47} \mathrm{As}$ layer is effective in making the surface smooth, and the thick GaSb buffer layer is effective in reducing the propagation of threading dislocations into the SL. ${ }^{(10)}$ The doping density in the GaSb buffer layer is $2.0 \times 10^{18} \mathrm{~cm}^{-3}$. InAs/GaSb SL, which consists of 100 pairs of 3.7-nm-thick InAs and 2.1-nm-thick GaSb, was grown on the GaSb buffer layer. To obtain a p-i-n structure, the GaSb layers in the bottom 30 pairs of $\mathrm{SL}$ were doped by Be. The doping density in the GaSb layers is $1.0 \times 10^{17} \mathrm{~cm}^{-3}$, and the InAs layers in the upper 30 pairs of SL were doped by Si. The doping density in the InAs layers is $2.0 \times 10^{18} \mathrm{~cm}^{-3}$. The middle 40 pairs were not intentionally doped. Finally, a Si-doped InAs cap layer of $20 \mathrm{~nm}$ thickness was grown on the SLs. The doping density in the InAs cap layer is $2.0 \times 10^{18} \mathrm{~cm}^{-3}$. As references, two types of InAs/GaSb SL were grown on Te-doped (100) GaSb substrates, where Be-doped GaSb buffer layers of 0.5 $\mu \mathrm{m}$ thickness and Si-doped InAs cap layers of $20 \mathrm{~nm}$ thickness were also grown. One SL consists of 100 pairs of 3.4-nm-thick InAs and 2.1-nm-thick GaSb and the other consists of 100 pairs of 3.9-nm-thick InAs and 2.1-nm-thick GaSb. The doping densities in the SLs, the buffer layers and the cap layers were the same as those on the InP substrate.

The crystalline quality of the epitaxial wafers was characterized by X-ray diffraction with $\mathrm{Cu} \mathrm{K} \alpha_{1}(\lambda=1.54056 \AA)$ and photoluminescence (PL) using a YAG laser (1064 nm) for the excitation source and an $\mathrm{HgCdTe}$ detector for the detection.

Mid-infrared PDs on the InP substrate were fabricated. The structure is illustrated in Fig. 2. The PDs were isolated by mesa etching using a solution including phosphoric acid $\left(\mathrm{H}_{3} \mathrm{PO}_{4}\right)$, citric acid $\left(\mathrm{C}_{6} \mathrm{H}_{8} \mathrm{O}_{7}\right)$, hydrogen peroxide $\left(\mathrm{H}_{2} \mathrm{O}_{2}\right)$ and water $\left(\mathrm{H}_{2} \mathrm{O}\right)$. The mesa diameters were changed from 30 to $920 \mu \mathrm{m}$. The sidewalls were passivated with a $\mathrm{SiO}_{2}$ layer deposited by plasma-enhanced chemical vapor deposition. Ti/Pt/Au electrodes were evaporated on both Si-doped InAs cap layers and Be-doped GaSb buffer layers, which were revealed by mesa etching. PDs on the GaSb substrates were also fabricated by the same procedure. 


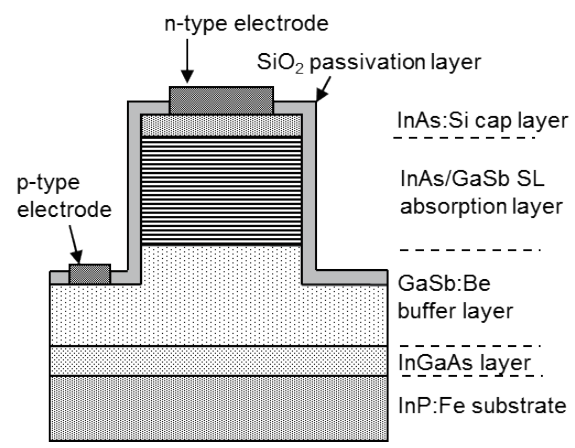

Fig. 2. Structure of PD on InP substrate.

Current-voltage characteristics of PDs were measured using a low-temperature wafer probing system with a semiconductor device analyzer. The quantum efficiencies were measured at $30 \mathrm{~K}$ and $-20 \mathrm{mV}$ bias with a monochromator using a diffraction grating. To estimate the absolute power of the measurement system, a thermopile detector (Dexter Model 2M) was used as a reference detector.

\section{Results and Discussion}

$\mathrm{X}$-ray rocking curves (XRCs) of the SLs on the InP and GaSb substrates (InAs thickness $3.9 \mathrm{~nm}$ ) are shown in Fig. 3. Satellite peaks are clearly seen in the XRC of the $\mathrm{SL}$ on the InP substrate, indicating that a periodic structure was realized, although they are wider than those of the SL on the GaSb substrate. In both XRCs, the peak positions of the SLs seem to coincide with those of the GaSb buffer layers, probably owing to the incorporation of $\mathrm{Sb}$ in the InAs layers.

PL spectra of the SLs on the InP and GaSb substrates were measured at $10 \mathrm{~K}$ (Fig. 4). The PL peak wavelengths were changed by the different thicknesses of InAs, and that of the SL on the InP substrate was $7 \mu \mathrm{m}$. The SL with 3.9-nm-thick InAs layers on the GaSb substrate exhibited a weaker PL peak than the SL with 3.4-nm-thick InAs layers on the GaSb substrate, which suggests that thicker InAs layers induce less overlap between wavefunctions of electrons and holes. On the other hand, the PL peak intensity of the SL on the InP substrate almost equals that of the SL with 3.4-nm-thick InAs layers on the GaSb substrate despite the thicker InAs layers. The strong PL intensity of the SL on the InP substrate seems to be contradictory to the fact that threading dislocations are found to be generated at the initial stage of the GaSb buffer layer growth and propagate to the SL in a previous study. ${ }^{(10)}$ The reason is not clear yet. However, the authors suppose that the strong excitation density $\left(7076 \mathrm{~W} / \mathrm{cm}^{2}\right)$ weakens the influence of the threading dislocations. Moreover, the high resistivity of the Fe-doped InP substrate might prevent the photogenerated carriers from diffusing into the substrate.

Current-voltage characteristics of the PDs on the $\mathrm{InP}$ and $\mathrm{GaSb}$ substrates (InAs thickness $3.9 \mathrm{~nm}$ ) at several temperatures were compared (Fig. 5). The low current densities of the PD on the GaSb substrate at forward bias seem to be derived from the 

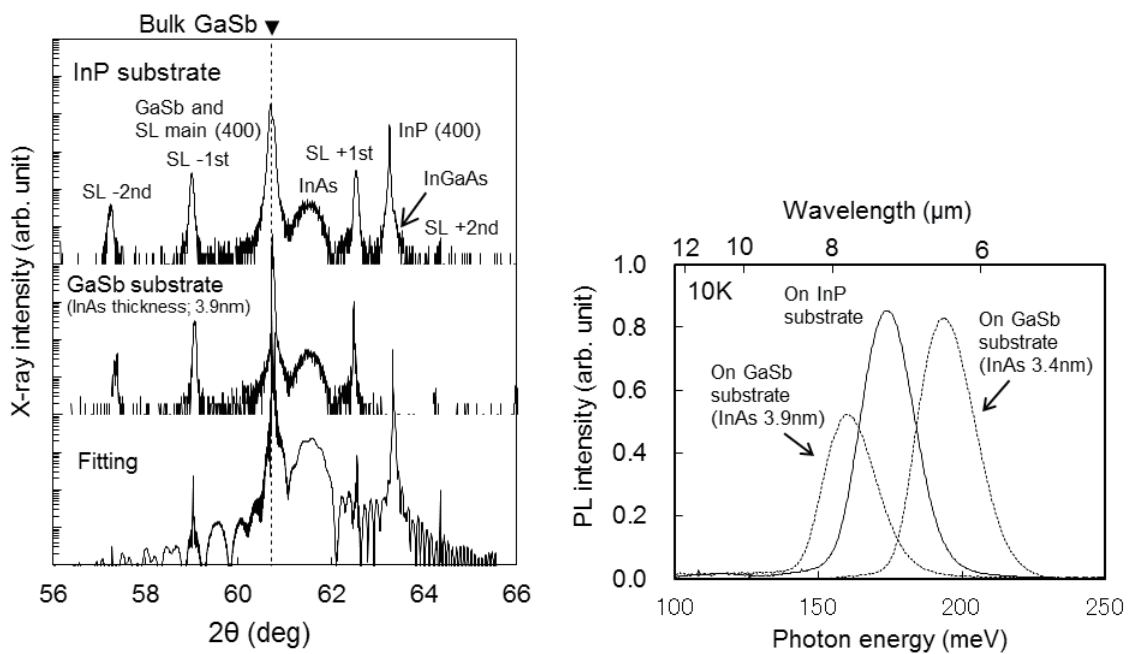

Fig. 3 (left). XRCs of SLs on InP and GaSb substrates.

Fig. 4 (right). PL spectra of SLs on InP and GaSb substrates.

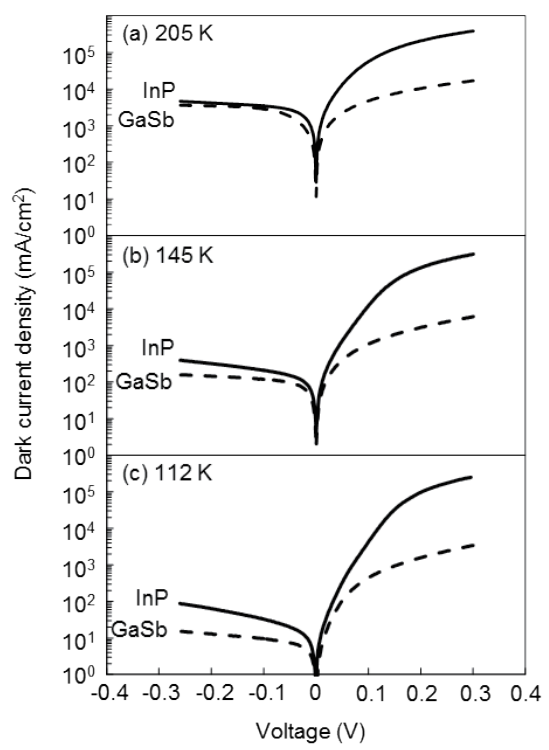

Fig. 5. Current-voltage characteristics of PDs on InP and GaSb substrates (The PD on the GaSb substrate contains 3.9-nm-thick InAs layers).

high device resistance owing to the thin Be-doped GaSb buffer layer. The ideal factor of the PD on the InP substrate calculated from the current-voltage characteristics at $112 \mathrm{~K}$ was 1.7 , which indicates that the current in the PD on the InP substrate is mainly composed of generation-recombination current rather than diffusion current. At reverse 
bias, the dark current of the PD on the InP substrate is comparable to that on the GaSb substrate at higher temperature. However, the separation of the dark current densities becomes more noticeable at a lower temperature. The temperature dependence of dark current densities at the reverse bias of $-0.1 \mathrm{~V}$ is shown in Fig. 6. The dark current densities increase exponentially as the inverse of temperature decreases (i.e., temperature increases). The factors $n$ of the PDs on the InP and GaSb substrates were calculated using the equation

$$
J \propto \exp \left(-E_{\mathrm{g}} / n k T\right)
$$

where $J, E_{\mathrm{g}}, k$, and $T$ stand for dark current density, band gap of the absorption layer, Boltzmann constant and temperature, respectively. The ideal value of factor $n$ is 1 , and it increases in accordance with the generation-recombination process via defects. ${ }^{(13)}$ The factors $n$ were 1.8 and 1.3 for the PDs on the InP and GaSb substrates, respectively. This result also suggests that the generation-recombination current is dominant in the PD on the InP substrate. In a previous study, threading dislocations are found to be generated at the initial stage of the GaSb buffer layer growth and propagate to the SL. ${ }^{(10)}$ It is inferred that the dark current of the PD on the InP substrate is generated by the threading dislocations. Suppression of threading dislocation in the SLs is necessary.

The external quantum efficiency spectra of the PDs were measured. The PD on the InP substrate showed an external quantum efficiency of about $10 \%$ around the wavelength of $5 \mu \mathrm{m}$ (Fig. 7). This value was comparable to those of the PDs on GaSb substrates. This result shows that the quantum efficiency was not sensitive to the threading dislocations, which is in contrast to the result of the dark current. It is reported that the quantum efficiency increases proportionally to the thickness of the SL absorption layer. ${ }^{(14)}$ It is expected that a thicker absorption layer would improve the quantum efficiency.
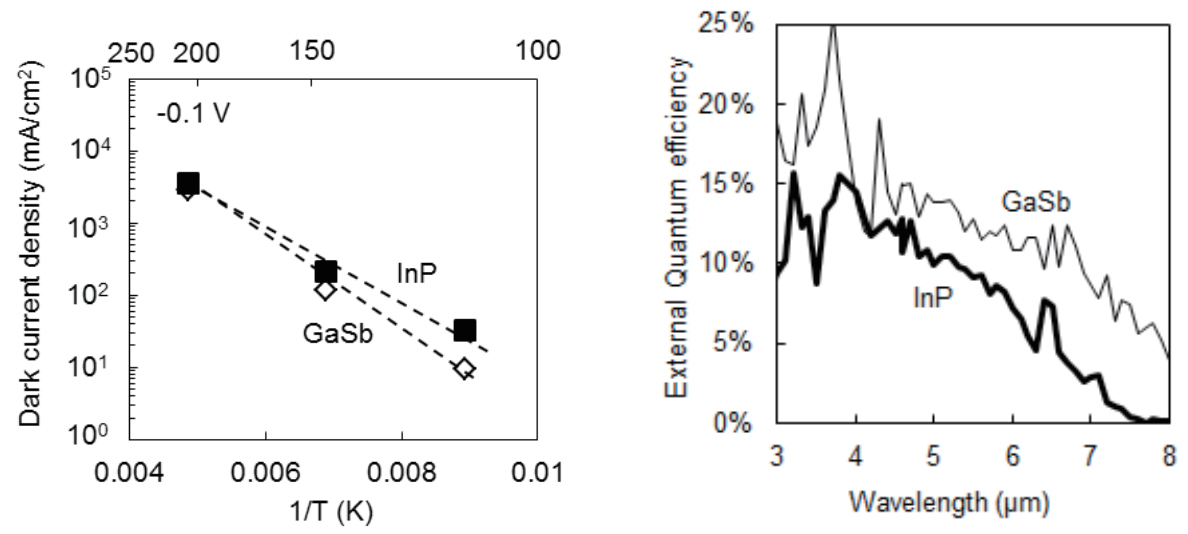

Fig. 6 (left). Temperature dependence of dark current densities of PDs on InP and GaSb substrates (The PD on the GaSb substrate contains 3.9-nm-thick InAs layers).

Fig. 7 (right). External quantum efficiency spectra of PDs on InP and GaSb substrates (The PD on the GaSb substrate contains 3.9-nm-thick InAs layers). 


\section{Conclusions}

Mid-infrared PDs with the type-II InAs/GaSb SL absorption layer grown on InP substrate were demonstrated. Although the dark current of the PD on the InP substrate is higher than that of the PD on the GaSb substrate, the external quantum efficiency of $10 \%$ was comparable to that on the GaSb substrate. The InAs/GaSb SLs grown on InP substrates have the potential to serve as the absorption layer of mid-infrared PDs.

\section{References}

1 S. D. Das, S. L. Tan, S. Zhang, Y. L. Goh, C. H. Tan and J. David: Proc. 6th EMRS DTC Technical Conference (2009) B7.

2 J. Bajaj, G. Sullivan, D. Lee, E. Aifer and M. Razeghi: Proc. SPIE 6542 (2007) 65420B.

3 M. Walther, J. Schmitz, R. Rehm, S. Kopta, F. Fuchs, J. Fleisner, W. Cabanski and J. Ziegler: J. Cryst. Growth 278 (2005) 156.

4 H. S. Kim, E. Plis, J. B. Rodriguez, G. D. Bishop, Y. D. Sharma, L. R. Dawson, S. Krishna, J. Bundas, R. Cook, D. Burrows, R. Dennis, K. Patnaude, A. Reisinger and M. Sundaram: Appl. Phys. Lett. 92 (2008) 183502.

5 P.-Y. Delaunay, B. M. Nguyen, D. Hoffman and M. Razeghi: Proc. SPIE 6542 (2007) 654204.

6 J. Chen, Y. Zhou, Z. Xu, J. Xu, Q Xu, H. Chen and L. He: J. Cryst. Growth 378 (2013) 596.

7 B.-M. Nguyen, D. Hoffman, E. K. Huang, S. Bogdanov, P.-Y. Delaunay, M. Razeghi and M. Z. Tidrow: Appl. Phys. Lett. 94 (2009) 223506.

8 B. Brar and D. Leonard: Appl. Phys. Lett. 66 (1995) 463.

9 P. M. Thibado, B. R. Bennett, M. E. Twigg, B. V. Shanabrook and L. J. Whitman: J. Vac. Sci. Technol. A14 (1996) 885.

10 K. Miura, Y. Iguchi and Y. Kawamura: J. Cryst. Growth 378 (2013) 121.

11 I. Vurgaftman, J. R. Meyer and L. R. Ram-Mohan: J. Appl. Phys. 89 (2001) 5815.

12 W. M. Yim and R. J. Paff: J. Appl. Phys. 45 (1974) 1456

13 H. Inada, K. Miura, H. Mori, Y. Nagai, Y. Iguchi and Y. Kawamura: Proc. SPIE 7660 (2010) $76603 \mathrm{~N}$.

14 A. Hood, D. Hoffman, B.-M. Nguyen, P.-Y. Delaunay, E. Michel and M. Razeghi: Appl. Phys. Lett. 89 (2006) 093506. 